Supplementary Information

\title{
Revealing an internal stabilization deficiency in the DNA polymerase $\beta$ K289M cancer variant through combined use of chemical biology and X-ray crystallography
}

\author{
Vinod K. Batra ${ }^{1}$, Khadijeh S. Alnajjar ${ }^{2}$, Joann B. Sweasy ${ }^{2}$, Charles E. McKenna ${ }^{3}$, Myron \\ F. Goodman ${ }^{4}$, and Samuel H. Wilson ${ }^{1 *}$
}

1. Genome Integrity and Structural Biology Laboratory, National Institute of Environmental Health Sciences, National Institutes of Health,111 T.W. Alexander Drive, Research Triangle Park, NC, 27709.

2. Department of Therapeutic Radiology and Genetics, Yale University School of Medicine, New Haven, Connecticut 06520.

3. Department of Chemistry, University of Southern California, Los Angeles, California 90089-1062

4. Department of Biological Sciences, University of Southern California, Los Angeles, California 90089-0371

*Corresponding Author: wilson5@,niehs.nih.gov

\section{Materials and Methods:}

For comparison, crystals of the "hot-spot sequence" oligonucleotides were also obtained with wild-type polymerase $\beta$ as described for K289M mutant under the main materials and methods section.

The "hot-spot sequence" of the template strand (16-mer), primer strand (9-mer) and phosphorylated downstream primer strand (5-mer) were 5'-CCGAACAAGCATCAGC-3', 5'GCTGATGCT-3', and 5'-pTTCGG-3', respectively. These mixtures were further incubated with ddTTP resulting in 1-nt gapped oligonucleotides with $\mathrm{dC}$ as the templating base. Rest of the procedure for crystallization and data collection was similar to that as described under materials and methods section of the main manuscript. 
$\mathbf{A}$

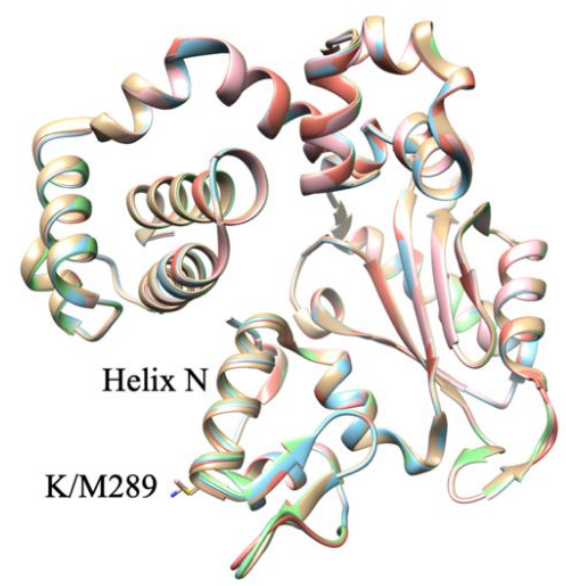

B

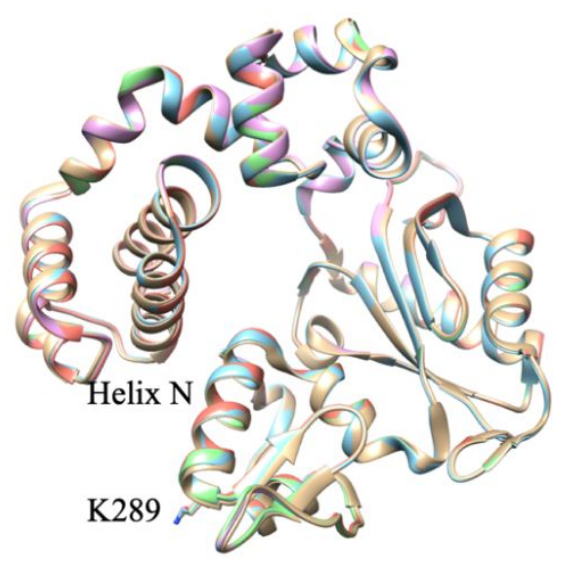

Figure S1: Overlay of ribbon diagrams of DNA polymerase $\beta$ wild-type and K289M structures with hot spot sequence and incoming dGTP analogues: The overlays were made with residues 10335 of the enzyme and with the wild-type enzyme in complex with the regular sequence (PBD 2FMS, gold).

A. K289M variant with hot-spot sequence and dGTP analogues: O(cyan), CHF (pink), $\mathrm{CH}_{2}$ (orange).

B. Wild-type enzyme with hot-spot sequence and dGTP analogues: O (cyan), CHF (pink), $\mathrm{CH}_{2}$ (orange). 
$\mathbf{0}$

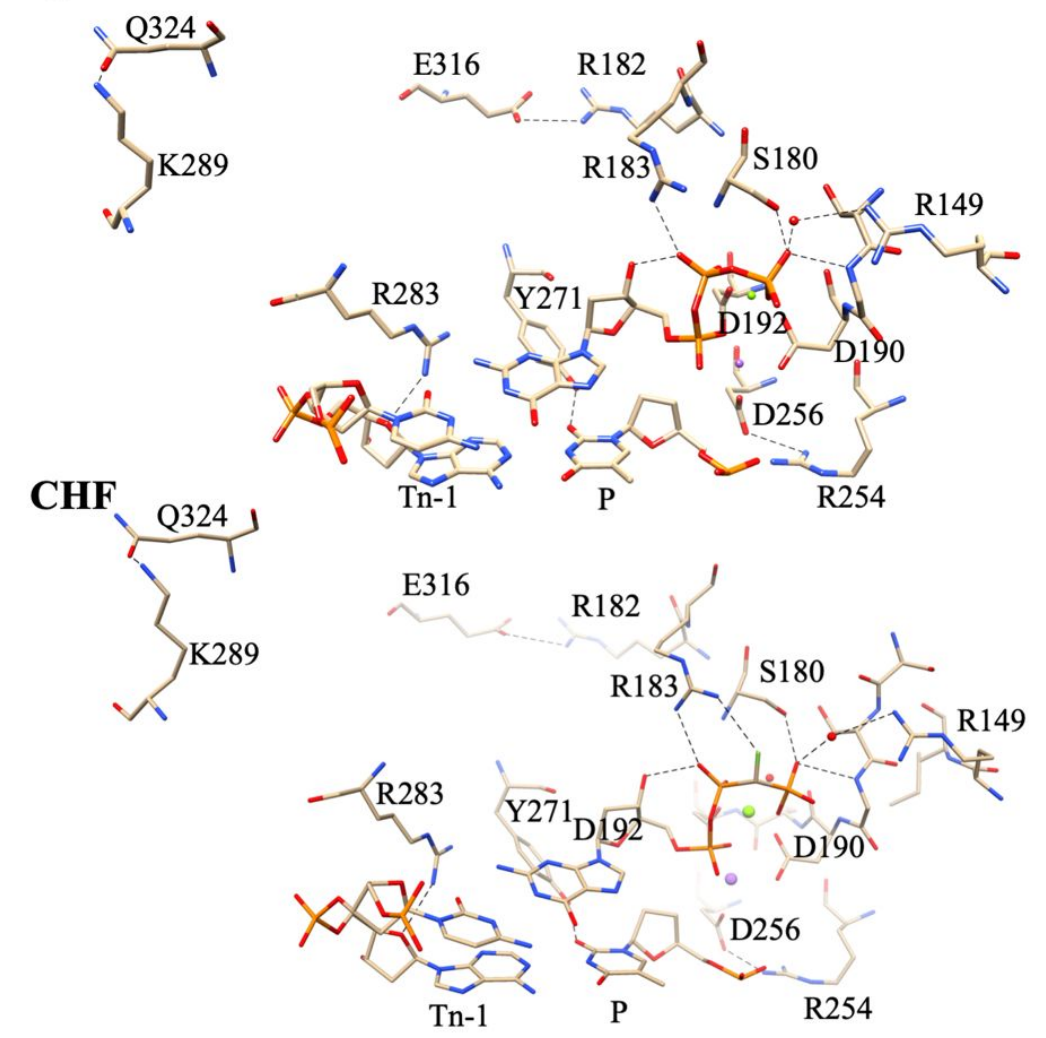

$\overbrace{K}^{\mathrm{CH}_{2}}$

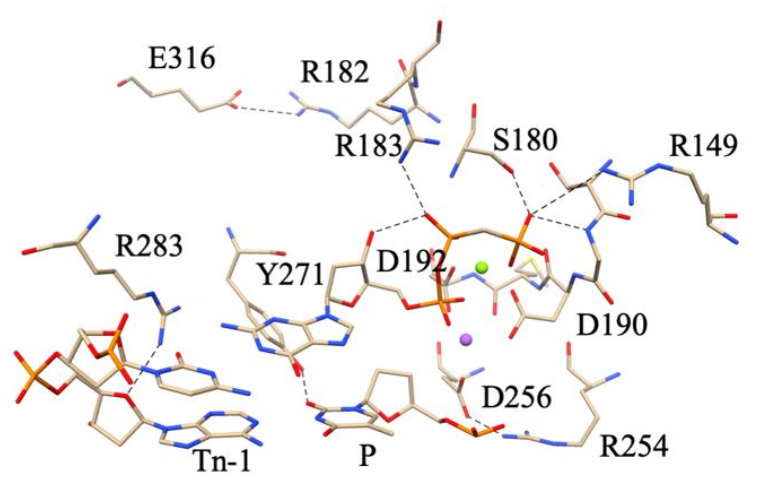

Figure S2: The active site of wild-type DNA polymerase $\beta$ with the incoming dGTP, O (top panel) or CHF (middle panel) or $\mathrm{CH}_{2}$ (bottom panel) analogue of dGTP with the "hot-spot sequence". All of these structures are similar to the reference structure (2FMS) with rmsd values of 0.240 to $0.314 \AA$. 

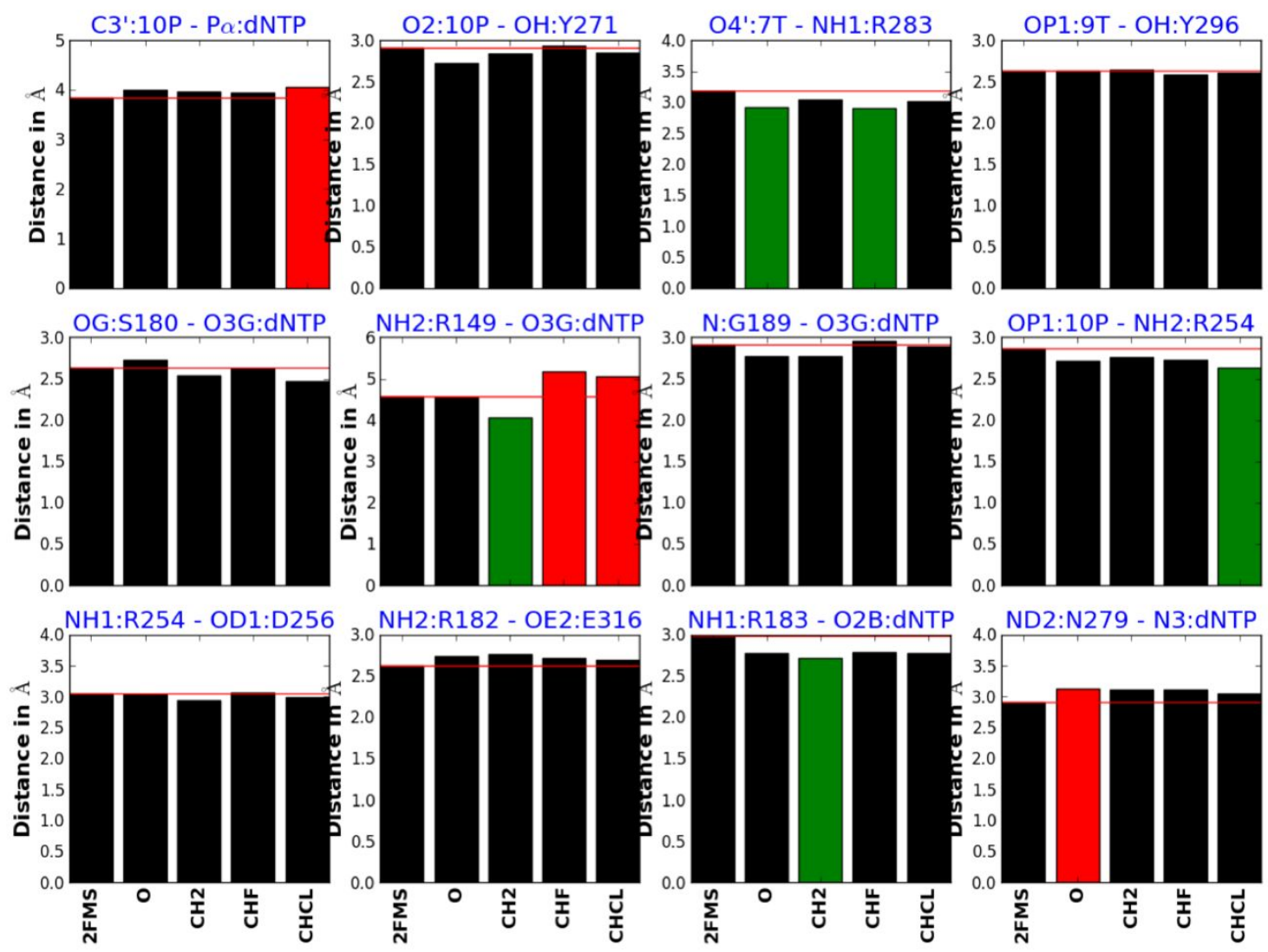

Figure S3: Bar diagram of main inter-atomic distances for dGTP analogues as shown for WTpolymerase $\beta$ with the "hot-spot sequence". The distances that are similar to those in the reference structures are colored black, the distances larger by $0.2 \AA$ are colored red, and the distances shorter by $0.2 \AA$ are colored green. 
$\mathbf{O}$

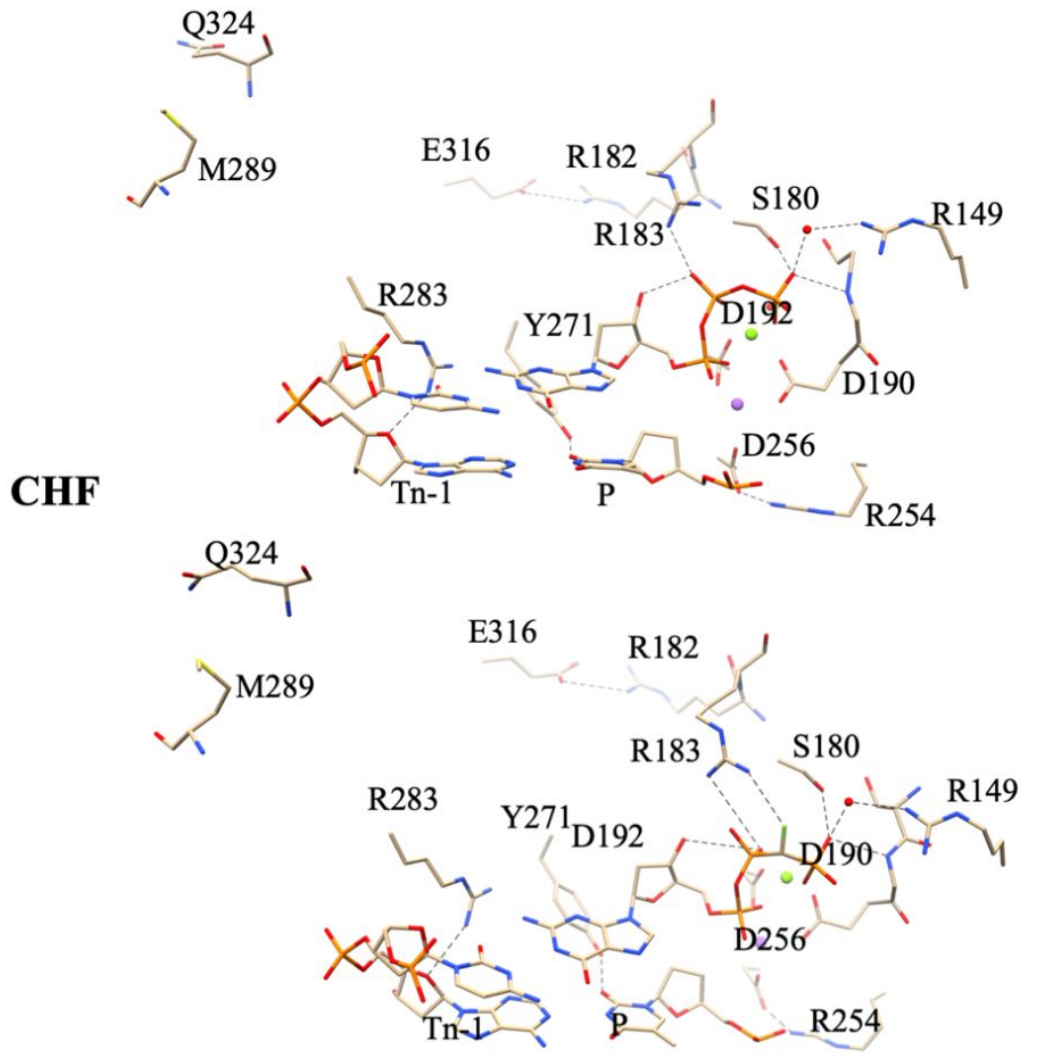

$\mathrm{CH}_{2} \overbrace{\text { Q324 }}^{\text {Q }}$

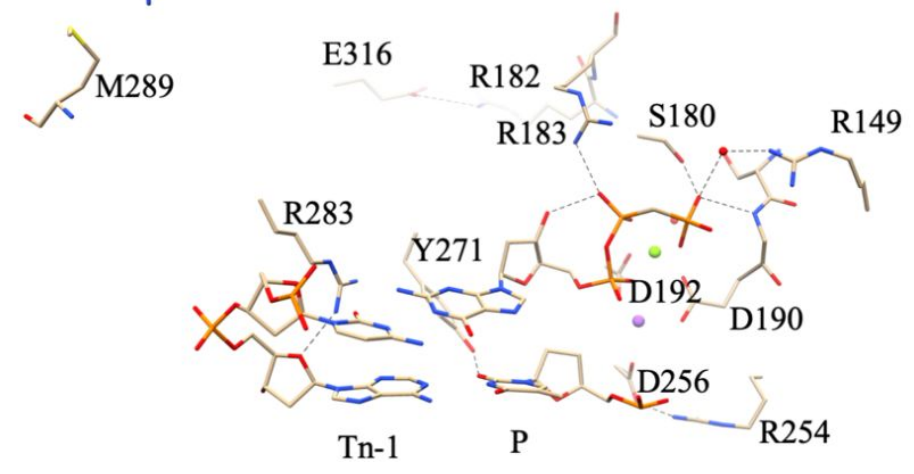

Figure S4: The active site of K289M variant with the incoming dGTP, O (top panel) or CHF (middle panel) or $\mathrm{CH}_{2}$ (bottom panel) analogue of dGTP with the "hot-spot sequence". The structures are similar to the reference structure (2FMS) with rmsd values of 0.208 to $0.310 \AA$. 


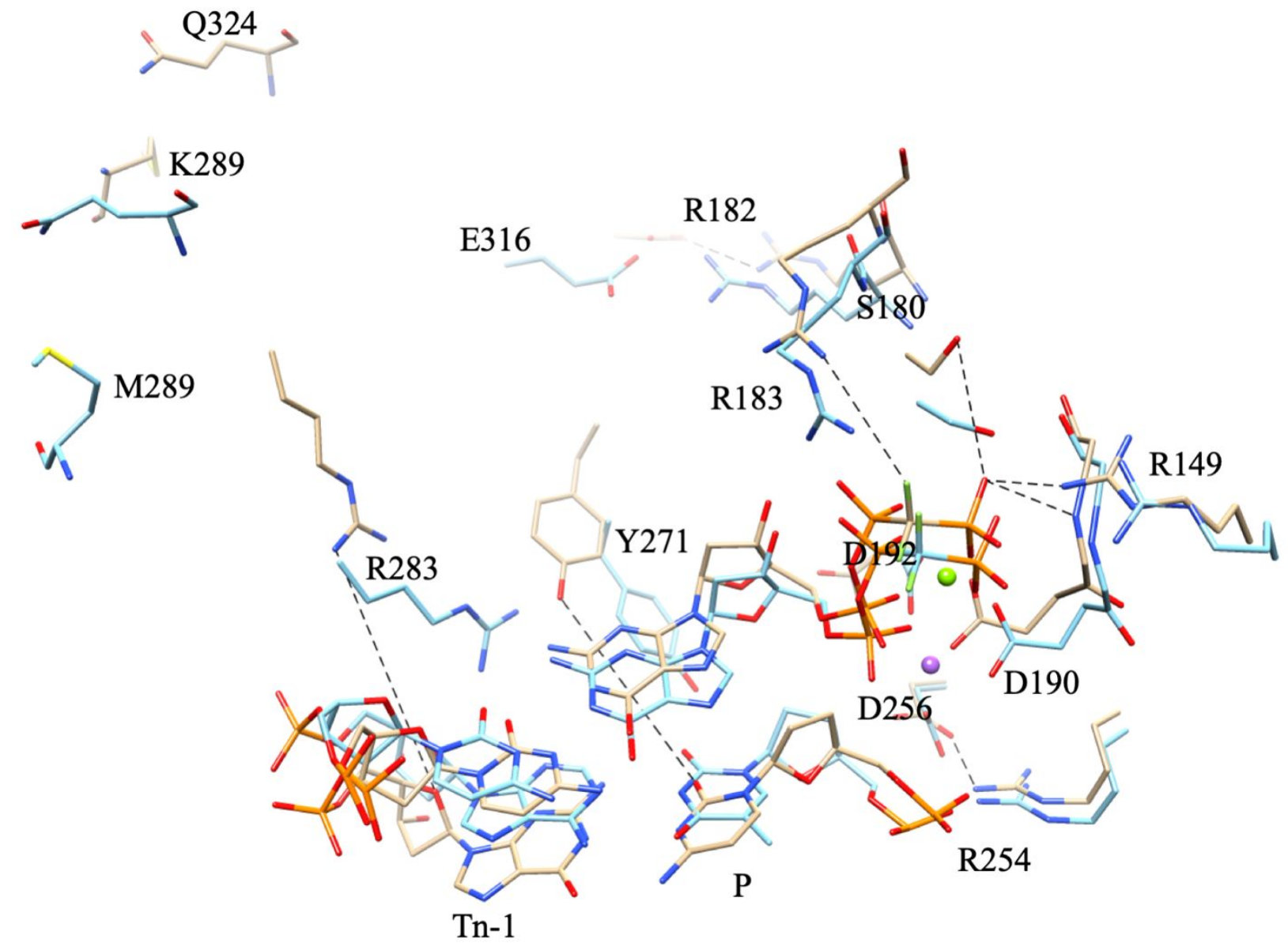

Figure S5: An overlay of the active site of K289M DNA polymerase $\beta$ with the incoming $\mathrm{CF}_{2}$ analogue of dGTP, comparing the regular sequence versus hot-spot sequence. 

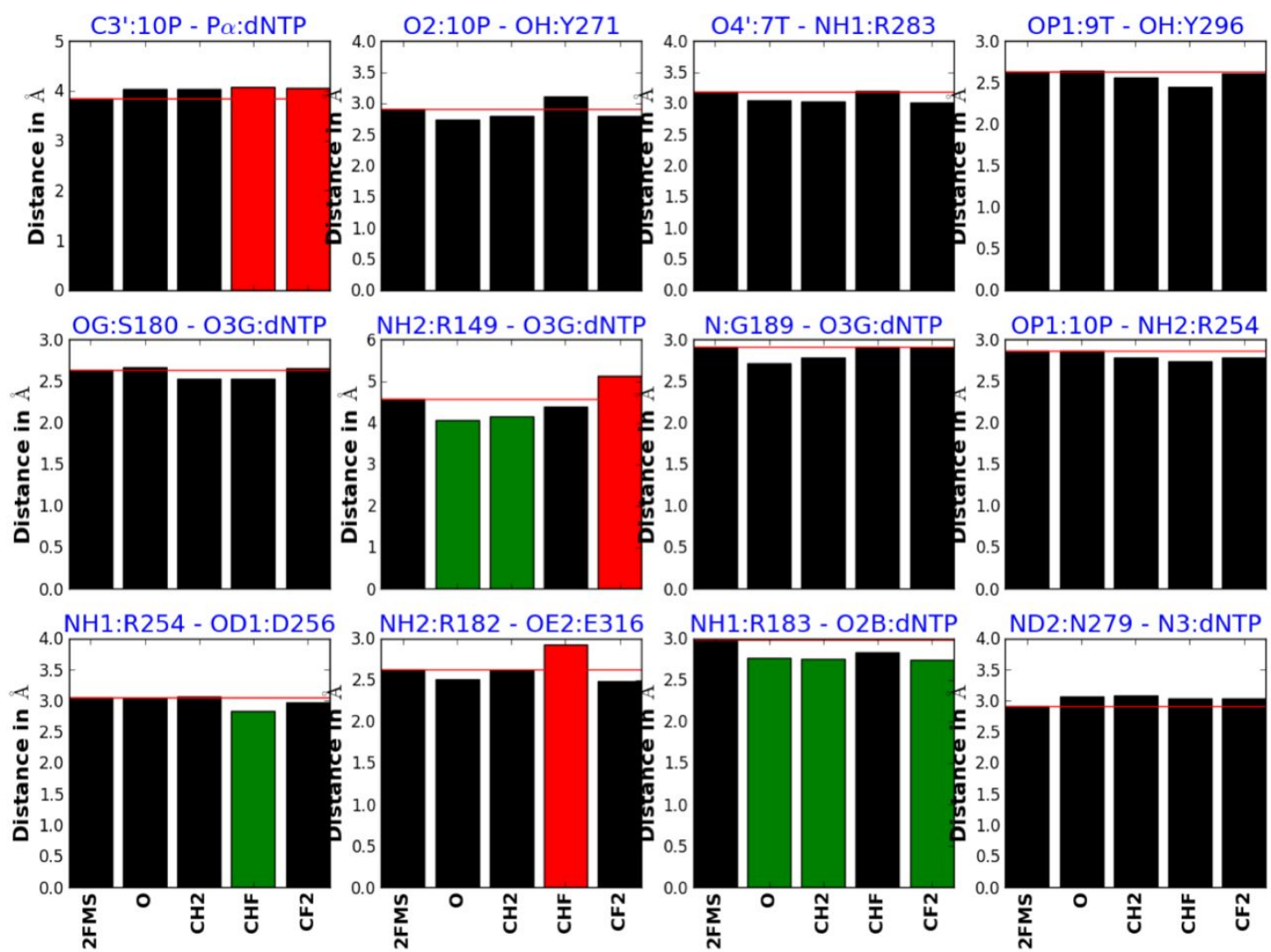

Figure 6: Bar diagram of main inter-atomic distances for dGTP analogues as shown for K289M variant with the "hot-spot sequence". The distances that are similar to those in the reference structures are colored black, the distances larger by $0.2 \AA$ are colored red, and the distances shorter by $0.2 \AA$ are colored green. 
Table S1: Data Collection and Refinement Statistics for K289M variant with regular sequence

\begin{tabular}{|c|c|c|c|}
\hline dGTP Analog & $\mathrm{O}$ & $\mathrm{CHF}$ & $\mathrm{CF}_{2}$ \\
\hline \multicolumn{4}{|l|}{ Data collection } \\
\hline Space group & $\mathrm{P} 2_{1}$ & $\mathrm{P} 2_{1}$ & $\mathrm{P} 2_{1}$ \\
\hline \multicolumn{4}{|l|}{ Cell dimensions } \\
\hline$a, b, c(\AA)$ & $50.5,80.1,55.3$ & $50.6,80.3,55.4$ & $55.3,79.7,54.7$ \\
\hline$\alpha, \beta, \gamma\left({ }^{\circ}\right)$ & $90,108.1,90$ & $90,108.1,90$ & $90,106.4,90$ \\
\hline Resolution $(\AA)$ & $50-2.45(2.49-2.45)^{*}$ & $50-2.35(2.39-2.35)$ & $50-2.30(2.34-2.30)^{*}$ \\
\hline$R_{\text {sym }}$ or $R_{\text {merge }}$ & $0.073(0.728)$ & $0.069(0.502)$ & $0.092(0.792)$ \\
\hline$I / \sigma I$ & $11.1(1.3)$ & $9.0(1.8)$ & $8.2(1.2)$ \\
\hline Completeness (\%) & $99.5(94.5)$ & $99.8(98.3)$ & $99.7(97.4)$ \\
\hline Redundancy & $3.6(2.8)$ & $3.6(3.1)$ & $3.3(2.0)$ \\
\hline \multicolumn{4}{|l|}{ Refinement } \\
\hline Resolution $(\AA)$ & 2.45 & 2.35 & 2.60 \\
\hline No. reflections & 55431 & 63077 & 52986 \\
\hline$R_{\text {work }} / R_{\text {free }}$ & $20.7 / 29.0$ & $18.9 / 25.5$ & $20.0 / 30.6$ \\
\hline \multicolumn{4}{|l|}{ No. atoms } \\
\hline Protein & 2603 & 2603 & 2564 \\
\hline Ligand/ion & 665 & 667 & 663 \\
\hline Water & 67 & 67 & 41 \\
\hline \multicolumn{4}{|l|}{$B$-factors } \\
\hline Protein & 50.1 & 40.8 & 46.1 \\
\hline Ligand/ion & 53.9 & 46.8 & 45.5 \\
\hline Water & 46.0 & 38.1 & 39.0 \\
\hline \multicolumn{4}{|l|}{ R.m.s. deviations } \\
\hline Bond lengths $(\AA)$ & 0.009 & 0.008 & 0.011 \\
\hline Bond angles $\left({ }^{\circ}\right)$ & 1.045 & 1.065 & 1.299 \\
\hline
\end{tabular}

*Values in parentheses are for the highest-resolution shell. 
Table S2: Data Collection and Refinement Statistics for WT Pol $\beta$ with the Hot-spot sequence

\begin{tabular}{|c|c|c|c|c|}
\hline dGTP Analog & $\mathrm{O}$ & $\mathrm{CH}_{2}$ & $\mathrm{CHF}$ & $\mathrm{CHCl}$ \\
\hline \multicolumn{5}{|l|}{ Data collection } \\
\hline Space group & $P 2_{1}$ & $P 2_{1}$ & $P 2_{1}$ & $P 2_{1}$ \\
\hline \multicolumn{5}{|l|}{ Cell dimensions } \\
\hline$a, b, c(\AA)$ & $50.6,79.8,55.4$ & $50.4,79.2,55.4$ & $50.6,79.7,55.4$ & $50.6,79.9,55.4$ \\
\hline$\alpha, \beta, \gamma\left({ }^{\circ}\right)$ & $90,107.7,90$ & $90,107.6,90$ & $90,107.96,90$ & $90,107.7,90$ \\
\hline Resolution $(\AA)$ & $\begin{array}{c}50-1.90 \\
(1.93-1.90)^{*}\end{array}$ & $\begin{array}{c}50-1.98 \\
(2.01-1.98) \\
\end{array}$ & $\begin{array}{l}50-1.85 \\
(1.88-1.85) \\
\end{array}$ & $\begin{array}{c}50-2.00 \\
(2.03-2.00) \\
\end{array}$ \\
\hline$R_{\text {sym }}$ or $R_{\text {merge }}$ & $0.046(0.316)$ & $0.070(0.420)$ & $0.065(0.208)$ & $0.053(0.322)$ \\
\hline$I / \sigma I$ & $19.3(2.2)$ & $11.3(1.8)$ & $17.8(5.3)$ & $12.4(2.0)$ \\
\hline Completeness (\%) & $90.3(52.3)$ & 97.1(92.9) & $89.7(46.4)$ & $93.1(77.3)$ \\
\hline Redundancy & $3.2(2.3)$ & $3.0(1.8)$ & $3.7(3.1)$ & $3.0(2.0)$ \\
\hline \multicolumn{5}{|l|}{ Refinement } \\
\hline Resolution $(\AA)$ & 1.90 & 1.98 & 1.85 & 2.00 \\
\hline No. reflections & 94751 & 85795 & 119833 & 79105 \\
\hline$R_{\text {work }} / R_{\text {free }}$ & $17.4 / 21.1$ & $18.6 / 23.5$ & $16.4 / 20.3$ & $17.8 / 22.1$ \\
\hline \multicolumn{5}{|l|}{ No. atoms } \\
\hline Protein & 2633 & 2632 & 2625 & 2622 \\
\hline Ligand/ion & 668 & 668 & 668 & 669 \\
\hline Water & 394 & 427 & 553 & 393 \\
\hline \multicolumn{5}{|l|}{$B$-factors } \\
\hline Protein & 29.9 & 26.0 & 21.1 & 28.3 \\
\hline Ligand/ion & 37.6 & 32.0 & 28.4 & 35.5 \\
\hline Water & 37.9 & 33.6 & 31.5 & 35.3 \\
\hline \multicolumn{5}{|l|}{ r.m.s. deviations } \\
\hline Bond lengths $(\AA)$ & 0.007 & 0.008 & 0.008 & 0.007 \\
\hline Bond angles $\left(^{\circ}\right)$ & 0.894 & 0.979 & 0.919 & 0.920 \\
\hline
\end{tabular}

*Values in parentheses are for the highest-resolution shell. 
Table S3: Data Collection and Refinement Statistics for K289M variant with Hot-spot sequence

\begin{tabular}{|c|c|c|c|c|}
\hline dGTP Analog & $\mathrm{O}$ & $\mathrm{CH}_{2}$ & $\mathrm{CHF}$ & $\mathrm{CF}_{2}$ \\
\hline \multicolumn{5}{|l|}{ Data collection } \\
\hline Space group & $P 2_{1}$ & $P 2_{1}$ & $P 2_{1}$ & $P 2_{1}$ \\
\hline \multicolumn{5}{|l|}{ Cell dimensions } \\
\hline$a, b, c(\AA)$ & $50.5,79.9,55.2$ & $\begin{array}{l}50.6,80.0, \\
55.2\end{array}$ & $50.6,80.2,55.3$ & $50.3,79.9,55.1$ \\
\hline$\alpha, \beta, \gamma\left({ }^{\circ}\right)$ & $90,108.0,90$ & $90,107.9,90$ & $90,107.9,90$ & $90,107.8,90$ \\
\hline Resolution $(\AA)$ & $\begin{array}{c}50-1.98 \\
(2.01-1.98)^{*}\end{array}$ & $\begin{array}{r}50-2.01 \\
(2.04-2.01)^{*}\end{array}$ & $\begin{array}{l}50-2.10 \\
(2.14-2.10)\end{array}$ & $\begin{array}{c}50-1.97 \\
(2.00-1.97)^{*}\end{array}$ \\
\hline$R_{\text {sym }}$ or $R_{\text {merge }}$ & $0.067(0.503)$ & $0.071(0.439)$ & $0.065(0.208)$ & $0.045(0.442)$ \\
\hline$I / \sigma I$ & $11.5(1.6)$ & $9.6(1.5)$ & $15(2.2)$ & $14.9(1.7)$ \\
\hline Completeness $(\%)$ & $96.4(65.9)$ & $96.5(63.9)$ & $92.2(41.2)$ & $96.5(76.4)$ \\
\hline Redundancy & $3.0(1.6)$ & $3.4(2.0)$ & $2.9(1.6)$ & $3.6(2.4)$ \\
\hline \multicolumn{5}{|l|}{ Refinement } \\
\hline Resolution $(\AA)$ & 1.98 & 2.01 & 2.10 & 1.97 \\
\hline No. reflections & 84760 & 91527 & 66796 & 103402 \\
\hline$R_{\text {work }} / R_{\text {free }}$ & $18.1 / 23.2$ & $18.6 / 23.5$ & $20.4 / 25.6$ & $19.3 / 23.7$ \\
\hline \multicolumn{5}{|l|}{ No. atoms } \\
\hline Protein & 2630 & 2613 & 2619 & 2619 \\
\hline Ligand/ion & 664 & 664 & 667 & 666 \\
\hline Water & 302 & 320 & 241 & 233 \\
\hline \multicolumn{5}{|l|}{$B$-factors } \\
\hline Protein & 30.4 & 32.4 & 40.0 & 39.4 \\
\hline Ligand/ion & 34.0 & 37.2 & 43.6 & 42.7 \\
\hline Water & 35.6 & 37.6 & 44.1 & 41.9 \\
\hline \multicolumn{5}{|l|}{ r.m.s. deviations } \\
\hline Bond lengths $(\AA)$ & 0.008 & 0.008 & 0.008 & 0.008 \\
\hline Bond angles $\left({ }^{\circ}\right)$ & 0.945 & 0.980 & 0.945 & 0.957 \\
\hline
\end{tabular}

*Values in parentheses are for the highest-resolution shell. 